\title{
Comparison of one-dimensional and three-dimensional models for the energy accommodation coefficient
}

\author{
Ali Özer, J.R. Manson * \\ Department of Physics and Astronomy, Clemson University, Clemson, SC 29634, USA
}

\begin{abstract}
The accommodation coefficient (AC) for the transfer of energy at a gas-surface interface is treated with a onedimensional (1D) theoretical model for classical scattering that retains full temperature dependence of both the gas and surface. Calculations are carried out for a purely repulsive gas-surface interaction and for the case in which there is an attractive adsorption well. Comparison with 3D calculations and with data for the accommodation of the rare gases at a tungsten surface indicates that full 3D dynamics are important for calculating the AC. (c) 2002 Elsevier Science B.V. All rights reserved.
\end{abstract}

Keywords: Atom-solid interactions; Noble gases; Tungsten

Since its initial introduction by Maxwell [1] one of the major parameters for describing energy exchange at a gas-surface interface is the energy accommodation coefficient (AC). The foundations for both theory and experimental measurements of the AC were established early in this century by Knudsen [2]. Several excellent reviews have covered continuing work on measuring and calculating the $\mathrm{AC}[3,4]$, and new treatments continue right up to the present [5].

Many previous theoretical treatments of the AC have been carried out using reduced dimensionality scattering models, in many cases one-dimensional (1D) models, or have needed to make other severe approximations such as setting the surface temperature equal to zero $[4,6]$. Recently, a fully $3 \mathrm{D}$ model with complete temperature dependence

\footnotetext{
* Corresponding author. Fax: +1-864-656-0805.

E-mail address: jmanson@ces.clemson.edu (J.R. Manson).
}

of both surface and gas has been applied to classical calculations of the AC [7]. The scattering model used has the distinct advantage, as compared to older treatments of the AC, that it has been demonstrated to describe state-to-state atom-surface scattering experiments for lowenergy ions or rare gases colliding with a variety of surfaces [8-10].

The purpose of this paper is to compare AC calculations made with 1D models to those made with full 3D scattering geometry. In particular, a scattering model is used that has explained the energy resolved spectra for the scattering of monoenergetic beams of rare gases from metal surfaces. This is the discrete model, one that treats the surface as a collection of discrete scattering centers. Of the several realistic classical scattering models available [11] only the discrete model admits to a straightforward reduction to $1 \mathrm{D}$.

It has been shown that calculations of the AC with the $3 \mathrm{D}$ discrete model underestimate the 
known experimental values for the heavy rare gases exchanging energy with a tungsten surface. A more sophisticated 3D model, which includes an attractive potential well and correct momentum conservation at the surface, was found to satisfactorily explain the same data [7].

However, in the present work it is found that the 1D model, in contrast to the 3D model, severely overestimates the AC. Improvements to the 1D model, such as adding an attractive potential well and including multiple collisions with the surface for the particles trapped in the well, further increases the AC and makes the agreement with measurements even worse.

Because of the generality of the discrete model, and the fact that it contains physically correct thermodynamics for both the surface and the gas, its failure appears to be a general result of 1D models. The basic conclusion to be drawn from this work is that full 3D scattering dynamics and correct conservation of momentum is essential for correctly describing thermal accommodation.

The energy $\mathrm{AC} \alpha_{\mathrm{E}}\left(T_{\mathrm{S}}, T_{\mathrm{G}}\right)$ is a measure of the energy actually exchanged by a gas at temperature $T_{\mathrm{G}}$ in contact with a surface at temperature $T_{\mathrm{S}}$ normalized to the maximum that could be exchanged if, on average, the gas atoms come into equilibrium with the surface after colliding with it. For a $1 \mathrm{D}$ system $\alpha_{\mathrm{E}}\left(T_{\mathrm{S}}, T_{\mathrm{G}}\right)$ is given by

$\alpha_{\mathrm{E}}\left(T_{\mathrm{S}}, T_{\mathrm{G}}\right)=\frac{\overline{E_{\mathrm{f}}}-k_{\mathrm{B}} T_{\mathrm{G}}}{k_{\mathrm{B}} T_{\mathrm{S}}-k_{\mathrm{B}} T_{\mathrm{G}}}$,

where $\overline{E_{\mathrm{f}}}$ is the average energy of a gas atom after colliding with the surface and $k_{\mathrm{B}}$ is the Boltzmann constant. It is convenient to define the equilibrium accommodation coefficient (EAC) $\alpha(T)$ as the limit of Eq. (1) in which the two temperatures become equal, i.e., in the $\lim \left\{T_{\mathrm{G}} \rightarrow T_{\mathrm{S}} \rightarrow T\right\}$. It is $\alpha(T)$ that will be of interest in this work.

The average final energy required for Eq. (1) is given by

$$
\begin{aligned}
\overline{E_{\mathrm{f}}}= & \int_{0}^{\infty} \mathrm{d} E_{\mathrm{i}} \int_{0}^{\infty} \mathrm{d} E_{\mathrm{f}} E_{\mathrm{f}} \frac{\mathrm{d} P^{\mathrm{MB}}\left(E_{\mathrm{i}}, T_{\mathrm{G}}\right)}{\mathrm{d} E_{\mathrm{i}}} \\
& \times \frac{\mathrm{d} R\left(E_{\mathrm{f}}, E_{\mathrm{i}}, T_{\mathrm{S}}\right)}{\mathrm{d} E_{\mathrm{f}}},
\end{aligned}
$$

where for a $1 \mathrm{D}$ system $\mathrm{d} R\left(E_{\mathrm{f}}, E_{\mathrm{i}}, T_{\mathrm{S}}\right) / \mathrm{d} E_{\mathrm{f}}$ is the differential reflection coefficient giving the proba- bility per unit final energy that an incident particle of energy $E_{\mathrm{i}}$ will be scattered with final energy $E_{\mathrm{f}}$, and $\mathrm{d} P^{\mathrm{MB}}\left(E_{\mathrm{i}}, T_{\mathrm{G}}\right) / \mathrm{d} E_{\mathrm{i}}=\exp \left\{-E_{\mathrm{i}} / k_{\mathrm{B}} T_{\mathrm{G}}\right\} / k_{\mathrm{B}} T_{\mathrm{G}}$ is the $1 \mathrm{D}$ Maxwell-Boltzmann distribution with streaming correction which gives the flux of incident atoms striking the surface.

The differential reflection coefficient must obey the condition of detailed balancing, given by

$$
\frac{\mathrm{d} R\left(E_{\mathrm{i}}, E_{\mathrm{f}}, T_{\mathrm{S}}\right)}{\mathrm{d} E_{\mathrm{i}}}=\exp \left(\frac{E_{\mathrm{f}}-E_{\mathrm{i}}}{k_{\mathrm{B}} T_{\mathrm{S}}}\right) \frac{\mathrm{d} R\left(E_{\mathrm{f}}, E_{\mathrm{i}}, T_{\mathrm{S}}\right)}{\mathrm{d} E_{\mathrm{f}}},
$$

i.e., Eq. (3) is the relation between scattering intensities for the two cases in which a particle scatters from energy $E_{\mathrm{i}}$ to $E_{\mathrm{f}}$, or scatters from the initial energy $E_{\mathrm{f}}$ to the final energy $E_{\mathrm{i}}$. Making use of the detailed balancing condition, the limit $T_{\mathrm{G}} \rightarrow T_{\mathrm{S}}$ can be carried out on Eq. (1), and after some manipulation a general expression for the $\mathrm{EAC}$ is given by

$$
\begin{aligned}
\alpha(T)= & \frac{1}{2\left(k_{\mathrm{B}} T\right)^{2}} \int_{0}^{\infty} \mathrm{d} E_{\mathrm{i}} \int_{0}^{\infty} \mathrm{d} E_{\mathrm{f}}\left(E_{\mathrm{f}}-E_{\mathrm{i}}\right)^{2} \\
& \times \frac{\mathrm{d} P^{\mathrm{MB}}\left(E_{\mathrm{i}}, T\right)}{\mathrm{d} E_{\mathrm{i}}} \frac{\mathrm{d} R\left(E_{\mathrm{f}}, E_{\mathrm{i}}, T\right)}{\mathrm{d} E_{\mathrm{f}}} .
\end{aligned}
$$

The discrete scattering model that will be used with Eq. (4) to evaluate the EAC is obtained by treating the scattering of a projectile of mass $m$ colliding with a surface of hard core scattering centers of mass $M$ that are in thermodynamic equilibrium at the temperature $T_{\mathrm{S}}$ [12]. The kinematical conditions of conservation of energy and momentum are obeyed upon collision. In 1D the differential reflection coefficient becomes

$$
\begin{aligned}
\frac{\mathrm{d} R}{\mathrm{~d} E_{\mathrm{f}}}= & \frac{1}{N_{\mathrm{i}}}\left(\frac{1}{4 \pi k_{\mathrm{B}} T_{\mathrm{S}} \Delta E_{0}}\right)^{1 / 2} \\
& \times \exp \left\{-\frac{\left(E_{\mathrm{f}}-E_{\mathrm{i}}+\Delta E_{0}\right)^{2}}{4 k_{\mathrm{B}} T_{\mathrm{S}} \Delta E_{0}}\right\},
\end{aligned}
$$

where $\Delta E_{0}=\mu\left(\sqrt{E_{\mathrm{i}}}+\sqrt{E_{\mathrm{f}}}\right)^{2}$ is the recoil energy of a $1 \mathrm{D}$ collision and $\mu=m / M$. The normalization constant $N_{\mathrm{i}}$ assures that the differential reflection coefficient obeys unitarity, i.e., the integral of $\mathrm{d} R / \mathrm{d} E_{\mathrm{f}}$ over all final energies equals 1 .

Calculations of the EAC of Eq. (4) using the differential reflection coefficient of Eq. (5) show 


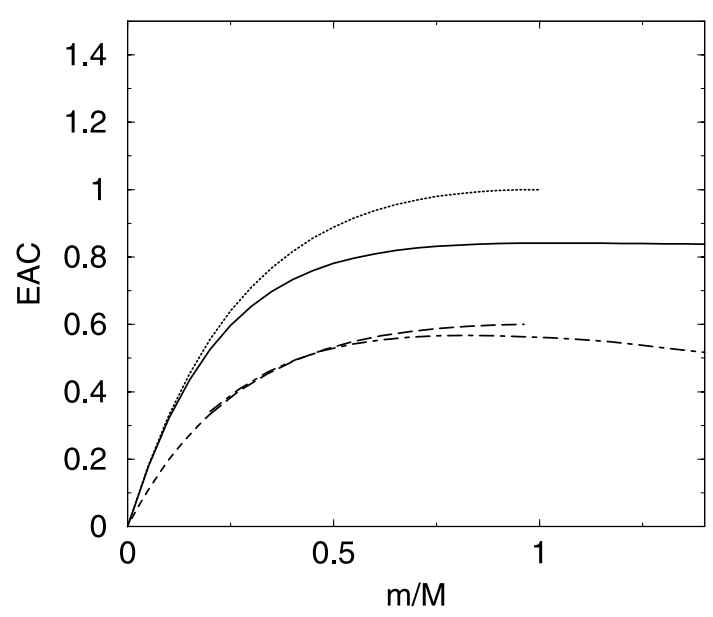

Fig. 1. The equilibrium energy $\mathrm{AC} \alpha(T)$ as a function of mass ratio $\mu=m / M$. The solid curve shows the 1D calculation of Eq. (4) and the dotted curve is the Baule formula of Eq. (6). The 3D calculation is the dash-dotted curve [7] and the dashed curve is Goodman's modified Baule formula of Eq. (7).

that $\alpha(T)$ is independent of the temperature and depends only on the mass ratio $\mu$. This dependence is shown in Fig. 1 where $\alpha(T)$ is plotted as a function of $\mu$ and compared with results of the same calculations for the full 3D discrete model. Also shown in Fig. 1 is the simple Baule expression for the $1 \mathrm{D} \mathrm{AC}$, which is the expression for the energy transfer in a head-on collision between two hard spheres

$\alpha=\frac{4 \mu}{(1+\mu)^{2}}$.

It is seen that the Baule approximation is in good agreement with the full calculations for $\mu<0.2$. The Baule approximation is valid only up to $\mu=1$, but the EAC calculated from Eq. (5) can be extended to $\mu>1$ because the thermal motion of the target can still back scatter the gas particles even if $m$ is greater than $M$.

The calculations for the 3D model involve integrations over incident and exiting angular variables in addition to the integrations over initial and final energies [7]. The results for this model are again independent of temperature and are also shown in Fig. 1 as a dash-dotted curve. Goodman has proposed a version of the Baule approximation of Eq. (6) $\alpha \approx \frac{2.4 \mu}{(1+\mu)^{2}}$,

which is obtained by averaging the Baule expression of Eq. (6) over classical trajectories in a 3D scattering process [13]. It is clear that the exact $3 \mathrm{D}$ calculations agree well with the Goodman expression for $\mu<0.6$. A significant point to be drawn from Fig. 1 is that the $1 \mathrm{D}$ calculation is much larger than the 3D calculation for all values of $\mu$. This is important because both are exact calculations carried out with the $1 \mathrm{D}$ and $3 \mathrm{D}$ versions of the same surface scattering model.

Up to this point the surface interaction has been treated with a purely repulsive potential. However, the Van der Waals forces between a surface and an approaching particle create an attractive well in front of the surface. The simplest way to introduce such an attractive interaction is with a square well potential. For classical scattering the primary effects of the adsorption well are to accelerate the incoming particle and cause it to collide with the surface at higher effective kinetic energy. A square well models this effect quite satisfactorily, not only under purely classical conditions, but also in atom-surface scattering under quantum mechanical conditions where it is known as the Beeby correction [14]. The approximation of using a square attractive well is further justified by its use in 3D calculations with models similar to that used here. A calculation and comparison with experimental data for the scattering of rare gases from liquid metals showed that a square well gave a good explanation of the effects of the attractive well on the observed scattered angular distributions [15].

The attractive well in the interaction potential will be important at low energies, where the energy is comparable to the well depth $D$. It also gives rise to additional phenomena, such as the possibility for sticking and trapping of gas atoms. For classical scattering the width of the well is unimportant. The effect on the scattering process is to enhance the energy at which the incoming gas particle collides with the repulsive wall according to

$E_{\mathrm{i}, \mathrm{f}}^{\prime}=E_{\mathrm{i}, \mathrm{f}}+|D|$. 
Once an attractive well is introduced, there is a non-zero probability that gas particles will become trapped in the well after a collision. Unless the surface temperature is very low, these trapped particles will suffer multiple collisions with the surface and eventually escape. When they escape, since they will have had many collisions inside the well, the appropriate assumption is that they will leave the surface with an equilibrium distribution at the temperature of the surface [4]. The fractional probability of trapped and scattered atoms is determined from the scattering kernel appearing in Eq. (4) according to

$$
\begin{aligned}
1= & \int_{0}^{\infty} \mathrm{d} E_{\mathrm{i}} \int_{0}^{\infty} \mathrm{d} E_{\mathrm{f}} \frac{\mathrm{d} P^{\mathrm{MB}}\left(E_{\mathrm{i}}, T_{\mathrm{G}}\right)}{\mathrm{d} E_{\mathrm{i}}} \frac{\mathrm{d} R^{\prime}\left(E_{\mathrm{f}}^{\prime}, E_{\mathrm{i}}^{\prime}, T_{\mathrm{S}}\right)}{\mathrm{d} E_{\mathrm{f}}^{\prime}} \\
& +\int_{0}^{\infty} \mathrm{d} E_{\mathrm{i}} \int_{-|D|}^{0} \mathrm{~d} E_{\mathrm{f}} \frac{\mathrm{d} P^{\mathrm{MB}}\left(E_{\mathrm{i}}, T_{\mathrm{G}}\right)}{\mathrm{d} E_{\mathrm{i}}} \frac{\mathrm{d} R^{\prime}\left(E_{\mathrm{f}}^{\prime}, E_{\mathrm{i}}^{\prime}, T_{\mathrm{S}}\right)}{\mathrm{d} E_{\mathrm{f}}^{\prime}} \\
= & N_{\mathrm{c}}+N_{\mathrm{t}} .
\end{aligned}
$$

In Eq. (9) $N_{\mathrm{c}}$ is the fraction of all atoms in the gas that are scattered back into the continuum after a collision. It is this contribution to the scattering kernel that is used to calculate the EAC from Eq. (4) in the presence of the well. Additionally, the trapped fraction $N_{\mathrm{t}}$ can be assumed to leave the surface eventually in an equilibrium distribution, thus this fraction is normally assigned an AC of unity and added to the contribution calculated from Eq. (4) using the fractional scattered probability [4].

For the fraction of gas particles that is trapped in the well after the first collision, the relatively simple model of a square well allows for following those particles as they continue to make successive collisions with the repulsive wall. The most physical approximation of this multiple scattering process is to assume that the trapped particles scatter elastically from the attractive step at the front of the well, and then scatter inelastically according to the differential reflection coefficient $\mathrm{d} R^{\prime}\left(E_{\mathrm{f}}^{\prime}, E_{\mathrm{i}}^{\prime}, T_{\mathrm{S}}\right) / \mathrm{d} E_{\mathrm{f}}^{\prime}$ of Eq. (5) evaluated at the repulsive wall inside the well. The justification that the attractive step of the square well does not vibrate, and hence does not contribute to inelastic scattering, is the same as the argument that the $V(z) \approx C_{3} / z^{3}$ surface Van der Waals potential is static, i.e., the Van der Waals attraction arises from a summation over the full 3D bulk of the target and hence all vibrational motion averages to zero. The process of multiple scattering in the well can be developed into an infinite series in which each successive term is a convolution of all previous scattering differential reflection coefficients. Such a series can be calculated exactly for a few low-order terms. Higher order terms can be summed by making use of several interesting approximations. Carrying out this multiple scattering process gives a more accurate evaluation than the simple assumption of an equilibrium distribution for the contribution to the differential reflection coefficient of the gas particles escaping from the trapped state after multiple scatterings. It also gives estimates of the lifetimes of particles in the trapped state.

A representative calculation for the EAC with an attractive well is shown in Fig. 2 for the accommodation of Ar gas at a tungsten surface. The $1 D$ EAC is plotted as a function of temperature $T$ with well depths $D=0 \mathrm{meV}$ (solid line) and $D=5$ meV (dashed curve). The data are those of Thomas [16] (open symbols) and of Kouptsidis and Menzel [17] (filled symbols). The general behavior

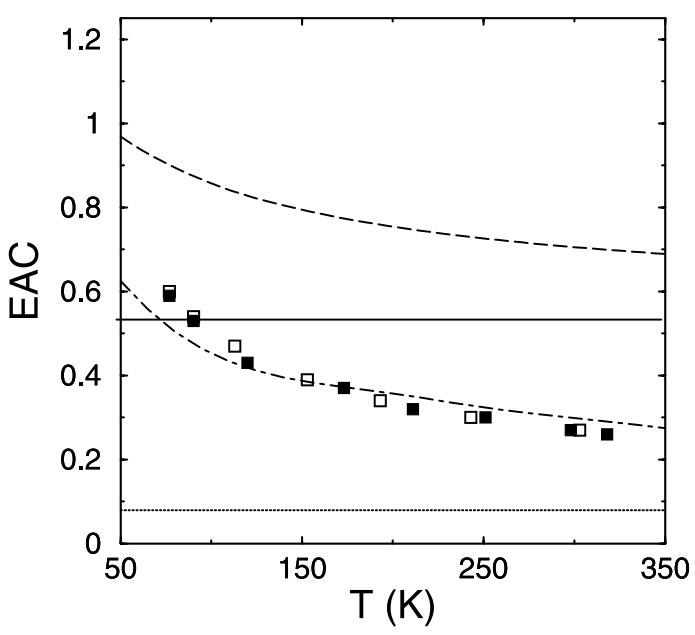

Fig. 2. The equilibrium energy $\mathrm{AC} \alpha_{\mathrm{E}}(T)$ as a function of surface temperature $T$ for Ar on a W surface. The experimental data are those of Thomas [16] ( $\square$ ) and of Kouptsidis and Menzel [17] (ם). The calculated curves for the 1D model are for well depth $D=0 \mathrm{meV}(-)$ and $D=5 \mathrm{meV}(---)$. The 3D model of Ref. [7] is shown for $D=0 \mathrm{meV}(\cdots)$ and $D=15 \mathrm{meV}$ $(-\cdot-)$. 
of $\alpha(T)$ as a function of $T$ and well depth $D$ is exhibited clearly here. Without a well, $\alpha(T)$ is essentially constant. With a well, the value of $\alpha(T)$ increases, with the increase becoming greater as $T$ decreases, in qualitative agreement with experiment. This general behavior of the EAC as a function of well depth is well known [4], and is similar for both 1D and full 3D calculations [7]. Also shown in Fig. 2 is the best fit 3D calculation using the smooth surface model discussed in Ref. [7] with $D=0 \mathrm{meV}$ (dotted line) and $D=15 \mathrm{meV}$ (dash-dot curve). The smooth surface model is one that assumes a smooth vibrating repulsive barrier for the atom-surface potential and includes the correct conservation of momentum, i.e., conservation of momentum only in directions parallel to the surface. For simplicity, the 3D discrete model calculations are not exhibited in Fig. 2, however, from Fig. 1 it is seen that for $D=0 \mathrm{meV}$ the AC value is constant and equal to 0.35 , while for $D=5 \mathrm{meV}$ it increases and is always larger than the experimental points but always significantly less than the $1 \mathrm{D}$ values.

The important point to be drawn from Fig. 2 is that the $1 \mathrm{D}$ calculation of the AC, even with a very small attractive well depth, not only overestimates the experimental values, but is also larger than the 3D discrete model calculations for the same well depth. This is in contrast to the case of full 3D calculations with the smooth surface model where, as seen in Fig. 2, it was possible to match the experimental data with a non-zero value of the well depth [7].

It should be noted that the best-fit value of $D=15 \mathrm{meV}$ for the well depth used in the calculations shown in Fig. 2 is small compared to measured and calculated values for this system. Although there is large discrepancy in the measured values, they range from $80 \mathrm{meV}$ to somewhat more than $120 \mathrm{meV}$ [18]. Theoretical calculations are somewhat smaller, ranging from 33 to $47 \mathrm{meV}$ [18].

The behavior of the EAC shown in Fig. 2 for Ar on tungsten is qualitatively the same in comparison with the experiments for the other heavy rare gases which have been measured, namely $\mathrm{Kr}$ and Xe. It should be mentioned that these calculations also overestimate the EAC for $\mathrm{He}$ and $\mathrm{Ne}$ accommodating at a tungsten surface. However, since $\mathrm{He}$ and $\mathrm{Ne}$ are light atoms they exhibit quantum behavior in surface scattering, and the present classical calculations are not expected to be valid for these gases at low temperatures.

To summarize, detailed calculations for a 1D model of the energy AC have been carried out, compared with previous 3D calculations, and compared with experimental data for rare gases accommodating at a tungsten surface. Classical surface scattering theory is adequate for the heavier rare gases and the discrete model was chosen for the following three reasons: (1) it contains the correct thermodynamics and full temperature dependence of both the gas and surface; (2) the discrete model is the appropriate 3D model from which one can reduce to the $1 \mathrm{D}$ case, and (3) the 3D discrete model is physically realistic because for several measured systems it has been demonstrated to explain state-to-state atom-surface scattering data.

The results of the calculations show that the EAC calculated with the 1D model is substantially larger than that calculated with the $3 \mathrm{D}$ version of the same model, and it severely overestimates the experimentally measured values. Improvements in the basic repulsive hard-core scattering, such as inclusion of an attractive well, increase the value of the calculated EAC and make the agreement with experiment even worse. On the other hand, a properly formulated $3 \mathrm{D}$ model can be made to agree with experiment.

Clearly, one conclusion that can be drawn from this work is that the 1D discrete model for atomsurface scattering is inadequate for calculations of the EAC because it consistently calculates values of the EAC that are too large. The fact that the 3D models can be made to agree with the experimental data with reasonable potential parameters indicates that full 3D scattering geometry and physically correct conservation of momentum parallel to the surface are important to correctly predict the accommodation at a gas-surface interface.

\section{Acknowledgements}

This work was supported by the US Department of Energy under grant number DE-FG02- 
98ER45704 and by the National Science Foundation under grant number DMR-0089503.

\section{References}

[1] J.C. Maxwell, Phil. Mag. 19 (1860) 20.

[2] M. Knudsen, The Kinetic Theory of Gases, Methuen, London, 1934.

[3] S.C. Saxena, R.K. Joshi, Thermal accommodation and adsorption coefficients of gases, in: C.Y. Ho (Ed.), CINDAS Data Series on Material Properties, Hemisphere Publishing Co., New York, 1989.

[4] F.O. Goodman, Prog. Surf. Sci. 5 (3) (1974) 261.

[5] M. Daw, submitted for publication.

[6] F.O. Goodman, H.Y. Wachman, Dynamics of GasSurface Scattering, Academic Press, New York, 1976.

[7] A. Muis, J.R. Manson, Surf. Sci. 486 (2001) 82.

[8] A. Muis, J.R. Manson, Phys. Rev. B 53 (1996) 2205.
[9] A. Muis, J.R. Manson, J. Chem. Phys. 108 (1997) 1655.

[10] M.F. Bertino, J.R. Manson, W. Silvestri, J. Chem. Phys. 108 (1998) 10239.

[11] J.R. Manson, Phys. Rev. B 58 (1998) 2253.

[12] A. Sjölander, Ark. Fys. 14 (1959) 315.

[13] F.O. Goodman, Surf. Sci. 7 (1967) 391.

[14] J.L. Beebe, J. Phys. C, Solid State Phys. 4 (1971) L359, 5 (1972) 3438.

[15] A. Muis, J.R. Manson, J. Chem. Phys. 111 (1999) 730.

[16] L.B. Thomas, in: C.L. Brundin (Ed.), Rarefied Gas Dynamics, Academic Press, New York, 1967, p. 155; L.B. Thomas, in: H. Saltzburg, J.N. Smith Jr., M. Rogers (Eds.), Fundamentals of Gas-Surface Interaction, Academic Press, New York, 1967, p. 346.

[17] J. Kouptsidis, D. Menzel, Berichte der Bunsen-Gesell, f. Phys. Chemie 74 (1970) 512;

J. Kouptsidis, D. Menzel, Zeits. f. Naturforsch. 24a (1969) 479.

[18] G. Vidali, G. Ihm, H.-Y. Kim, M. Cole, Surf. Sci. Rep. 12 (1991) 135. 